\section{Investigación cuantitativa: buscando la estandarización de un esquema taxonómico}

\section{Quantitative research: Seeking the standardization of a taxonomic scheme}

\section{Señor Editor,}

La Ley Universitaria 30.220 promulgada en el 2014 ha impulsado que la mayoría de las universidades peruanas entren en un proceso de transición y consolidación de sus instituciones en el ámbito científico ${ }^{1}$. Muchas escuelas profesionales de ciencias de la salud han incorporado asignaturas que mejoren los conocimientos y las competencias de sus estudiantes en la producción científica.

Actualmente, en los cursos de metodología de la investigación y afines, se utilizan esquemas sobre el diseño de investigación de enfoques cuantitativos que en ciertos casos han mostrado inconsistencias para los estudios realizados por los estudiantes. En múltiples casos se observa que ellos suelen forzar sus estudios para acoplarlos a dichos esquemas. Son varias las ocasiones donde los docentes mencionan que en dichos esquemas pueden haber "excepciones" donde los estudios planteados no encajan adecuadamente.

Por tal razón es necesario mostrar un esquema práctico que agrupe los principales criterios taxonómicos de investigación de enfoques cuantitativos tomando en referencia lo establecido por la Sociedad Hispana de Investigadores Científicos ${ }^{2}$. En la siguiente gráfica se muestran cuatro criterios que determinarán cuatro rasgos importantes a declarar sobre el diseño de las diferentes investigaciones: la intervención del investigador, la intención de análisis, los momentos de evaluación a las unidades de investigación y la naturaleza de los datos (Figura 1).

Los cuatro criterios trabajan de manera independiente, por lo que la opción escogida en cada uno de los criterios no influye en la selección de las opciones de los demás criterios.

Según la intervención del investigador, es decir, la existencia de cualquier grado de manipulación de cualquiera de las variables a medir, existen dos posibilidades: ser un estudio observacional si es que en ningún momento se realizó manipulación alguna de las variables, o ser experimental si se llegó a producir alguna manipulación ${ }^{2}$. Hernández Sampieri ${ }^{3}$ y múltiples autores desde comienzos de los años 60's han considerado este criterio como punto de partida a la hora de clasificar el diseño de investigación.

Según la intención analítica existen dos posibilidades: descriptivo si es que se realizó un análisis univariado en todo el procesamiento estadístico, o analítico si es que se realizó análisis multivariado al menos una vez durante dicho procesamiento. Es importante mencionar que los estudios descriptivos no necesariamente tienen una sola variable, sino que pueden tener una gran variedad de variables las cuales serían evaluadas de manera independiente 2 .

Según los momentos de recolección de datos se tiene dos posibilidades: transversal, si cada unidad de investigación es evaluada una única vez durante el proceso de investigación; o longitudinal, si se ha evaluado dos o más veces ${ }^{2}$.

Según la naturaleza de los datos se tiene dos extremos y una tercera opción que considera a ambos: retrospectivo, si los datos provienen de alguna base de datos, documentos o fuentes similares originados previo al proceso de recolección de datos (no hay control

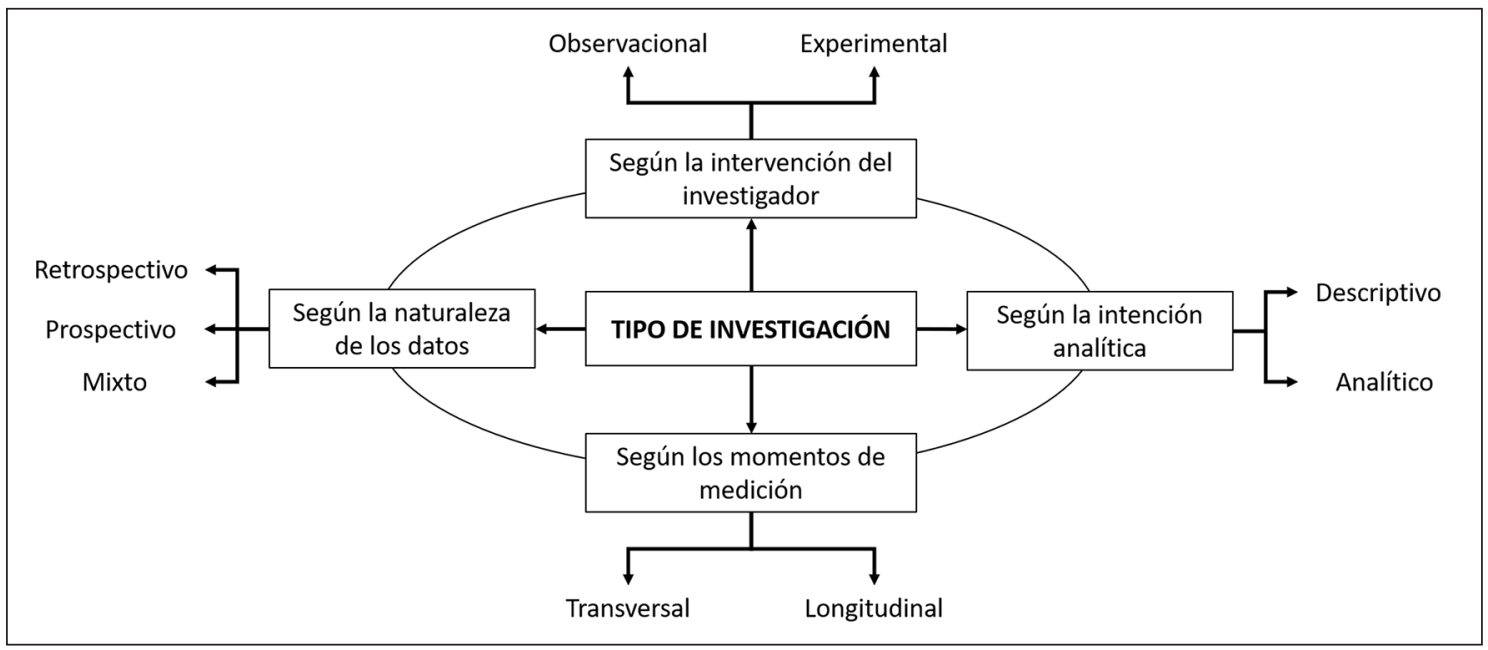

Figura 1. Clasificaciones de investigaciones cuantitativas. Fuente: Chacma-Lara E. 
sobre la generación de datos); prospectivo, si es que los datos se están creando a propósito de la investigación (hay control sobre la generación de datos); o mixto, si se utilizan datos ya existentes y a la vez se van a generar datos nuevos ${ }^{2}$. Algunos autores conceptualizan este criterio desde otra perspectiva tomando el aspecto de la lineabilidad temporal de los sucesos estudiados y consideran que los estudios retrospectivos, prospectivos y mixtos solo pueden ubicarse dentro de los estudios longitudinales ${ }^{4}$. Este último razonamiento es aún aceptado por una parte considerable de la comunidad científica; sin embargo, en este caso se excluye la posibilidad de mencionar una cualidad importante (naturaleza de los datos) de los estudios transversales, por lo que no se permite mencionar de forma práctica el control sobre la generación de los datos.

Se espera que este esquema sea de utilidad en la docencia con la finalidad de mejorar el entendimiento de la metodología de la investigación en cuanto al diseño de estudios cuantitativos; así como recomendar que todos los artículos científicos consideren mencionar estos cuatro criterios dentro de las secciones de materiales y métodos permitiendo una busca más eficiente en el momento de revisión de antecedentes.

\section{Edward Chacma-Lara ${ }^{1}$, Thirsa Laura-Chávez ${ }^{1}$ ${ }^{1}$ Escuela de Medicina Humana, Universidad Privada San Juan Bautista, Lima, Perú.}

\section{Referencias}

1. Congreso de la República del Perú. Ley Universitaria [Internet]. Ley No 30220 Perú: Poder Legislativo; 2014. Disponible en: http://www.minedu.gob.pe/reforma-universitaria/ pdf/ley_universitaria.pdf

2. Supo J. Metodología de la Investigación Científica. Lima, Perú: Sociedad Hispana de Investigadores Científicos; 2020. $352 \mathrm{p}$.

3. Sampieri RH, Fernández C, Baptista P. Metodología de la investigación [Internet]. 6th Ed. S.A. M-H/ IE, editor. Vol. 53. 2014. 1-589 p. Disponible en: https://mail.google.com/ mail/u/1/\#inbox/15a4cf4b02ab7f85?projector $=1$

4. Londoño F JL. Metodología de la investigación epidemiológica. 5th Ed. Editorial El Manual Moderno; 369d. C. 2014 p.

\section{Correspondencia a:}

Edward Silvestre Chacma Lara

Escuela de Medicina Humana

Universidad Privada San Juan Bautista

Av. Lavalle S/N., Chorrillos. Lima 09-Perú.

edward.chacma@gmail.com 